\title{
Yersinia enterocolitica Bloodstream Infection
}

\author{
Douglas W. Challener ${ }^{\mathrm{a}, \mathrm{c}}$, Eugene M. Tan ${ }^{\mathrm{b}}$, \\ John C. O’Horo ${ }^{\text {b }}$
}

\begin{abstract}
An elderly male presented with abdominal pain and constipation. He was diagnosed with Yersinia enterocolitica bloodstream infection associated with an abdominal aortic aneurysm and synthetic endovascular graft. This demonstrates a rare but known complication of $Y$. enterocolitica bloodstream infection.
\end{abstract}

Keywords: Yersinia enterocolitica; Bacteremia; Bloodstream infection

\section{Introduction}

Yersinia enterocolitica is a gram-negative organism found worldwide. It rarely causes bloodstream infection, and even more rarely is associated with mycotic aneurysm formation. Its association with aneurysm formation may not be recognized prior to endovascular repair resulting in possibility of a graft infection.

\section{Case Report}

A 72-year-old male presented to an emergency department with 2 weeks of abdominal pain and constipation. His relevant medical history included a history of splenectomy many years ago due to a motor vehicle accident, and many complications of atherosclerotic disease including symptomatic right carotid artery (RCA) stenosis status post endarterectomy and saphenous vein synthetic fabric patch graft approximately 10 years before his presentation.

The patient lived in rural Minnesota his whole life and had not traveled outside the state. He was retired but previously worked in a slaughterhouse. He consumed cooked pork on a

Manuscript submitted September 27, 2017, accepted October 12, 2017

aDepartment of Internal Medicine, Mayo Clinic, 200 First Street SW, Rochester, MN 55905, USA

bivision of Infectious Diseases, Mayo Clinic, 200 First Street S.W., Rochester, MN 55905, USA

${ }^{\mathrm{c} C}$ Corresponding Author: Douglas W. Challener, Department of Internal Medicine, Mayo Clinic, 200 First St S.W., Rochester, MN 55905, USA.

Email: challener.douglas@mayo.edu

doi: https://doi.org/10.14740/jmc2931w regular basis. He owned two healthy pet dogs.

The emergency department obtained a computed tomography $(\mathrm{CT})$ scan of the abdomen. It revealed a $5.1-\mathrm{cm}$ infrarenal abdominal aortic aneurysm as well as increased signal in the periaortic fat consistent with an early aortic leak. The outside hospital arranged his transfer to a higher level of care due to high concern that his abdominal pain was ischemic and possibly due to an abdominal aortic aneurysm (AAA) leak. The accepting facility repeated a $\mathrm{CT}$ angiogram, which demonstrated the AAA with associated soft tissue stranding concerning for impending rupture. The surgery team performed an emergent endovascular aortic stent graft. The day following the surgery, the lab detected $Y$. enterocolitica in one of three blood culture bottles (obtained before the surgery) at $28 \mathrm{~h}$. His team initiated vancomycin and piperacillin-tazobactam. Two days later, $Y$. enterocolitica grew from a subsequent, post-surgical blood culture in one of two sets. He was transitioned to ceftriaxone 2 $\mathrm{g}$ IV every $24 \mathrm{~h}$ with a plan to treat for 6 weeks and suppress with doxycycline indefinitely given the likelihood that he was bacteremic during his stent placement.

\section{Discussion}

Y. enterocolitica was first described in 1934 as the cause of a farmer's facial abscess by McIver and Pike [1]. Over the years, several names including Bacterium enterocoliticum and Flavobacterium pseudomallei have been used to describe this aerobic, gram-negative bacteria. This organism is thought to have reservoirs in many animals including pigs, cats, dogs, rabbits, and sheep. It does not appear to cause disease outside of humans [2].

$Y$. enterocolitica is an enteric pathogen and is transmitted most commonly via the fecal-oral route, usually through contaminated food or water. Blood transfusion can also transmit Y. enterocolitica [3]. There have been several major outbreaks in the United States, often related to milk. The US Department of Agriculture (USDA) attributed at least one outbreak to a homemade preparation of Chitterlings, a food made from the small intestines of pigs [3]. PCR of raw pork products in the United States has revealed high levels of these bacteria. One study found the stool prevalence of $Y$. enterocolitica in pigs in the Midwest and Eastern United States was 13.1\% [4]. A sampling of residents in Europe found serum antibodies present in $31 \%$ of residents in Finland and $43 \%$ of those sampled in Germany [5]. It seems to occur more frequently in countries with cooler climates [6].

The classical syndrome consists of gastrointestinal mani- 
festations of abdominal pain, fever, and an inflammatory, watery diarrhea, especially in children. Young adults often manifest with mesenteric lymphadenitis that can symptomatically mimic appendicitis [1]. The syndrome is usually self-limited and resolves within 2 - 3 weeks without treatment. Up to $15 \%$ of those infected experience a reactive polyarthritis, especially in patients with HLA-B27 [5].

Bloodstream infection is very uncommon and often fatal. In one large cohort from Belgium, only $0.5 \%$ of microbiologic isolates were from blood [7]. The elderly, immunocompromised, and patients with iron overload or on pharmacologic iron chelation are more likely to experience disseminated infection $[1,2]$. Investigators have cultured the bacteria from blood, CSF, urine, and the anterior chamber of the eye [8]. Y. enterocolitica has been implicated in the formation of mycotic aneurysms in the abdominal aorta as well as other vessels [9]. This is very rare with less than 20 cases reported in the literature [10]. Most of these cases are elderly, hypertensive males [11]. A previous research has associated the O9 serotype with vascular infections more than other serotypes [12].

This case is particularly interesting and unique because of the potential involvement of the synthetic endovascular graft placed during the bacteremia. A similar case of endovascular repair of a mycotic aneurysm caused by $Y$. enterocolitica exists in the literature. That endovascular repair was performed several days after initiation of antibiotics, and that patient received 8 weeks of post-operative antibiotics [10]. In this case, however, the graft was in place during the bacteremia, and duration of treatment was determined knowing that the graft was likely infected.

The bacteria have demonstrated in vitro susceptibilities to trimethoprim-sulfamethoxazole, aminoglycosides, tetracycline, chloramphenicol, piperacillin, ciprofloxacin, and third generation cephalosporins. Antibiotics typically show little benefit in enterocolitis but are indicated in bloodstream infections. There is not a standard antibiotic regimen in these cases, but most have success with the above antibiotics $[2,6]$.

While $Y$. enterocolitica bloodstream infections are a rarity, their involvement in a mycotic aneurysm is even rarer. While not formally demonstrated with histological or microbiological evaluation of the endograft, this case is likely one of the few cases of a graft infection by $Y$. enterocolitica. It shows the importance of blood cultures before and after placement of synthetic endovascular grafts. These cultures confirmed that the graft was placed during a bloodstream infection. This knowledge creates high concern that the graft itself was infected and would substantially alter the final therapeutic course.

\section{Funding}

No funding sources.

\section{References}

1. Bottone EJ. Yersinia enterocolitica: the charisma continues. Clin Microbiol Rev. 1997;10(2):257-276.

2. Mead PS. 231 - Yersinia Species (Including Plague). Eighth Edition. 2016:2607-2618.e2.

3. Bottone EJ. Yersinia enterocolitica: overview and epidemiologic correlates. Microbes Infect. 1999;1(4):323-333.

4. Bhaduri S, Wesley IV, Bush EJ. Prevalence of pathogenic Yersinia enterocolitica strains in pigs in the United States. Appl Environ Microbiol. 2005;71(11):7117-7121.

5. Fredriksson-Ahomaa M, Stolle A, Korkeala H. Molecular epidemiology of Yersinia enterocolitica infections. FEMS Immunol Med Microbiol. 2006;47(3):315-329.

6. Cover TL, Aber RC. Yersinia enterocolitica. N Engl J Med. 1989;321(1):16-24.

7. Van Steen J, Vercruysse J, Wilms G, Nevelsteen A. Arteriosclerotic abdominal aortic aneurysm infected with Yersinia enterocolitica. Rofo. 1989;151(5):625-626.

8. Sonnenwirth AC. Bacteremia with and without meningitis due to Yersinia enterocolitica, Edwardsiella tarda, Comamonas terrigena, and Pseudomonas maltophilia. Ann N Y Acad Sci. 1970;174(2):488-502.

9. Donald K, Woodson J, Hudson H, Menzoian JO. Multiple mycotic pseudoaneurysms due to Yersinia enterocolitica: report of a case and review of the literature. Ann Vasc Surg. 1996;10(6):573-577.

10. Zhang J, Miller S, Wilton G, Kumar P. Yersinia enterocolitica: a rare cause of infected aortic aneurysm successfully treated with antibiotics and endovascular repair. JMM Case Reports. 2014;1(2):1-4.

11. Hagensee ME. Mycotic aortic aneurysm due to Yersinia enterocolitica. Clin Infect Dis. 1994;19(4):801-802.

12. Prentice MB, Fortineau N, Lambert T, Voinnesson A, Cope D. Yersinia enterocolitica and mycotic aneurysm. Lancet. 1993;341(8859):1535-1536. 